Conclusion The first lockdown resulted in a decrease of $62.7 \%$ of infected individuals. A remarkable decrease of resistant gonococcal strains to azithromycin and no resistance to cefixime occurred in 2020. This is in contrast to an increase of resistances until 2019. Increasing numbers of isolates of women approached the MIC breakpoint for ceftriaxone.

\section{P351 CLINICAL PRESENTATIONS OF SYPHILIS DIAGNOSED AT WESTERN SYDNEY SEXUAL HEALTH CENTRE, 2015-2019}

${ }^{1} Y$ Hughes*, ${ }^{1} \mathrm{C}$ Chung, ${ }^{1,2,3} \mid$ Zablotska-Manos, ${ }^{1,2,3} \mathrm{D}$ Lewis. 'Western Sydney Sexual Health Centre, Parramatta, Australia; ${ }^{2}$ Westmead Clinical School, Westmead, Australia; ${ }^{3}$ Marie Bashir Institute for Infectious Diseases and Biosecurity, Westmead, Australia

\subsection{6/sextrans-2021-sti.398}

Background Correct syphilis staging requires attentive historytaking and clinical examination; this assists with optimal patient management. We describe the clinical presentations of syphilis cases diagnosed at Western Sydney Sexual Health Centre (WSSHC) over a five-year period.

Methods We undertook a retrospective descriptive study of syphilis presentations at WSSHC between January 2015 and December 2019. Paper and electronic medical records were reviewed for 557 syphilis cases managed during this period. Clinical data were extracted and entered into an Access database for subsequent analysis. The Chi square test for trend was used to analyse incident infections over time. Ethics Committee approval was obtained.

Results During the study period, there were 203 symptomatic (93 primary, 110 secondary) and 354 asymptomatic (351 latent, 3 late neurosyphilis) syphilis cases. Primary and secondary syphilis cases increased over time $(\mathrm{p}=0.038$ and $\mathrm{p}=0.004$, respectively). Most primary $(91 / 93,98 \%)$ and secondary $(99 / 110,90 \%)$ cases were in men. Ulceration was a feature of 92/93 (99\%) primary syphilis cases; one additional patient presented with isolated inguinal lymphadenopathy. Locations of syphilitic ulceration were as follows: penile (68, $74 \%)$, anal $(19,21 \%)$, male genital skin $(4,4 \%)$ and oral $(4$, $4 \%)$. Most ulcers were solitary $(65 / 93,70 \%)$ although $30 \%$ (27/92) were multiple. Most of the secondary syphilis cases presented with a rash $(84 / 110,76 \%)$; the rash was demonstrable on palms/soles in 34/84 (40\%) cases and on the trunk in $73 / 84(87 \%)$ cases. Palmoplantar rash alone occurred in $7 / 84$ $(8 \%)$. Oral lesions and condylomata lata were documented in $16(15 \%)$ and 10 (9\%) of secondary syphilis patients, respectively. Six (5\%) secondary syphilis patients also had CSF-confirmed neurosyphilis; three were symptomatic (headache, $n=2$; neck stiffness, $\mathrm{n}=1$; blurred vision/photophobia, $\mathrm{n}=1$; reduced hearing, $\mathrm{n}=1$ ).

Conclusions Syphilis presents in many different ways and correct staging requires careful examination in order to inform clinical management and partner notification practice.

\section{P354 LABORATORY CAPACITY ASSESSMENT FOR SEROLOGICAL DETECTION OF SYPHILIS IN GUANGDONG, CHINA}

1,2L Yiwen*, ${ }^{2} \mathrm{Q}$ Xiaolin, ${ }^{1,2} \mathrm{Z}$ Heping. ${ }^{1}$ Dermatology Hospital of Southern Medical University, Guangzhou, China; ${ }^{2}$ Southern Medical University, Guangzhou, China

10.1136/sextrans-2021-sti.399
Objective To investigate and identify the detection capability of syphilis in the STD (sexually transmitted disease) laboratories in Guangdong, China.

Method An External quality assessment (EQA) of syphilis tests was performed and an online questionnaire surveys on tests and screening algorithms were conducted. Three positive and two negative EQA panels were prepared for qualitative or quantitative test of Nontreponemal tests (NTT) and Treponemal test (TT). The samples were distributed to all participants in October 2019 and the results were reported within one month and analyzed by Guangdong Central STD Laboratory through an online quality assessment software.

Results A total of 838 laboratories participated in the program, which come from different STD clinics, including primary, secondary and tertiary general hospitals, Maternal and Child Health Care Hospitals and CDC from the provincial, prefecture and county levels in Guangdong. The results showed that overall coincidence rate of the 838 participated laboratories was $98.0 \%$. The coincidence rate of NTT were that $99.3 \%$ of qualitative test and $96.7 \%$ of quantitative test. Coincidence rate of qualitative TT were 99.3\%. There were 286 laboratories reported the quantitative TT results, with an overall coincidence rate of $94.7 \%$. Toluidine red unheated serum test (TRUST) was the most used NTT, and Treponema pallidum particle assay (TPPA) popularly chosen as Treponemal test. There were 273 laboratories returned a second survey on syphilis screening algorithms. Among them, 118(43.2\%) laboratories adopted the traditional screening, 81(29.7\%) laboratories adopted the reverse screening, and 64(23.4\%) laboratories employed two algorithms simultaneously. Moreover, 10(3.7\%) laboratories adopted the ECDC (European Centre for Disease Control and Prevention) algorithm.

Conclusion Laboratories in Guangdong Province have great capabilities of syphilis diagnosis, especially in the CDC and the Maternal and Child Hospitals. Traditional screening is the most used algorithm, while the use of reverse screening is increasing.

\section{P355 THE PERFORMANCE OF LABORATORY-BASED DIAGNOSTIC ASSAYS TO DIAGNOSE PRIMARY SYPHILIS CASES AT WESTERN SYDNEY SEXUAL HEALTH CENTRE, 2015-2019}

${ }^{1} Y$ Hughes* ${ }^{1}{ }^{1} \mathrm{C}$ Chung, ${ }^{1,2,3}$ Zablotska-Manos, ${ }^{1,2,3} \mathrm{D}$ Lewis. ${ }^{1}$ Western Sydney Sexual Health Centre, Parramatta, Australia; ${ }^{2}$ Westmead Clinical School, Westmead, Australia; ${ }^{3}$ Marie Bashir Institute for Infectious Diseases and Biosecurity, Westmead, Australia

\subsection{6/sextrans-2021-sti.400}

Background Treponema pallidum subsp. pallidum infection (TP) may be diagnosed either directly by dark-field microscopy (DFM) or polymerase chain reaction (PCR) assay, or indirectly by serology. TP PCR and serology are currently the mainstay of laboratory-based diagnosis due to technical challenges associated with DFM. We describe the performance of TP PCR and serology in the diagnosis of primary syphilis at Western Sydney Sexual Health Centre (WSSHC).

Methods This is a retrospective study of primary syphilis cases diagnosed at WSSHC over a five-year period (2015-2019). Medical case records were reviewed to extract laboratory results. Patients without a prior history of syphilis were screened with either an enzyme-linked immunosorbent total antibody assay (EIA, WSSHC-associated laboratory) or a 
chemiluminescent microparticle immunoassay (CMIA, private laboratories). The non-treponemal Venereal Disease Research Laboratory (VDRL) assay was used to screen patients previously-treated for syphilis. An in-house PCR assay tested ulcerderived DNA extracts for TP. The study received Ethics Committee approval.

Results 93 primary syphilis cases (92 with ulceration, 1 with inguinal lymphadenopathy) were diagnosed in the study period. TP PCR was performed in 84/92 (91\%) cases and positive in $76 / 84$ (90\%) cases. Overall, syphilis serology detected 79/93 (85\%) primary syphilis cases. 75/93 (81\%) cases had no laboratory evidence of prior syphilis; 18 cases (19\%) had previous reactive treponemal serology. Treponemal antibodies were present in 63/75 (84\%) first-episode syphilis cases; $12 / 75$ (16\%) had non-reactive treponemal serology. Most cases with previously-treated syphilis had reactive VDRL tests $(16 / 18,89 \%)$. There were 64 PCR positive/serology reactive cases, 12 TP PCR positive/serology non-reactive cases, 7 PCR negative/serology reactive cases and one clinically-diagnosed case (negative TP PCR/non-reactive serology). Serology was reactive in all 9 cases where TP PCR was not performed (8 EIA reactive, 1 VDRL reactive).

Conclusion Primary syphilis is best diagnosed using a combined approach with TP PCR and syphilis serology.

\section{P357 A NEW MEASURE OF SEXUAL WELLBEING FOR COMMUNITY SURVEYS: DEVELOPMENT AND VALIDATION OF THE THE NATSAL-SW}

${ }^{1} \mathrm{~K}$ Mitchell ${ }^{*},{ }^{2} \mathrm{M}$ Palmer, ${ }^{1} \mathrm{R}$ Lewis, ${ }^{1} \mathrm{R}$ Boso Perez, ${ }^{1} \mathrm{~K}$ Maxwell, ${ }^{2} \mathrm{~W}$ Macdowell, ${ }^{2} \mathrm{D}$ Reid, ${ }^{2} \mathrm{C}$ Bonell, ${ }^{3} \mathrm{P}$ Sonnenberg, ${ }^{3} \mathrm{C}$ Mercer, ${ }^{4} \mathrm{~J}$ Fortenberry. ${ }^{1}$ University of Glasgow, Glasgow, UK; ${ }^{2}$ London School of Hygiene and Tropical Medicine, London, UK; ${ }^{3}$ University College London, London, UK; ${ }^{4}$ Indiana University, Indianapolis, USA

\subsection{6/sextrans-2021-sti.401}

Background Sexual wellbeing is intrinsic to public health but long-standing conflation of sexual health and sexual wellbeing has limited our ability to address everyday sexual issues. This study proposed a seven-domain model, and developed and validated a brief measure for community surveys.

Method Domains of sexual wellbeing were determined through critical engagement with wide-ranging literature and 40 semi-structured interviews to explore resonance with lived experiences. Measure development involved 7 cognitive interviews and two web-based surveys of general population samples $(n=590, n=814)$, to assess performance of individual items, conduct exploratory and confirmatory factor analysis, and examine whether the resultant measure was associated with external variables as hypothesised. A sub-sample $(n=113)$ completed the survey again after two weeks to test re-test reliability.

Results We proposed seven domains of sexual wellbeing: security and safety; respect; self-esteem; resilience; forgiveness of past sexual experiences; self-determination and comfort. Semistructured interviews confirmed the relevance of these domains to lived experiences of sex and sexuality. Drawing on the semi-structured and cognitive interviews we drafted a 25-item measure to capture these domains. Based on individual item assessment and exploratory and confirmatory factor analyses, we trimmed the measure to 13 -items. The confirmatory factor analysis indicated that a 'general specific model' had best fit (RMSEA: 0.064; CFI: 0.975, TLI: 0.962), and functioned equivalently across age groups, genders, sexual orientation and relationship status. The final measure was associated with external variables in the directions hypothesised (all $\mathrm{p}<0.001$ ), including sexual functioning (coefficient $=0.924$ ), mental wellbeing (0.454), self-esteem (0.564), sexual esteem (0.563), body image $(0.232)$, depression $(-0.384)$, anxiety $(-0.340)$, sexual satisfaction (0.680) and sexual distress (-0.615) and demonstrated good test-retest reliability (ICC $=0.78$ ).

Conclusion Our conceptual model and 13-item measure distinguishes sexual wellbeing from sexual health and enables sexual wellbeing to be quantified and understood within and across populations.

\section{P358 ASSESSING SEXUAL HEALTH SERVICES AT A PUBLIC UNIVERSITY IN THE UNITED STATES}

A Camino*, M Whitfield, N Van Wagoner. University of Alabama At Birmingham School of Medicine, Birmingham, USA

\subsection{6/sextrans-2021-sti.402}

Background The American National College Health Association reports that college students have frequent, condomless sex. Student health and wellness clinics (SHWC) offer sexual health services, but few have dedicated sexual health clinics (SHC). We evaluated screening service use at a university SHWC after implementation of a SHC two half-days per week.

Methods This was a retrospective analysis of data from patients receiving sexual health screening at the University of Alabama at Birmingham (UAB) SHWC from 2015 to 2019. Demographic variables and rates of STIs were extracted from the electronic medical record and were compared by clinic (SHC vs. SHWC). Univariate models were fit, and multi-variable models will be fit, selecting variables with $\mathrm{p}$ values of 0.1 or less. Odds ratios with corresponding 95\% confidence intervals for univariate analysis are presented.

Results A total of 5025 STI screenings were performed. Males (OR 4.13; 3.61-4.72), undergraduates (OR 1.33; 1.15-1.54), and persons reporting sex with the same sex (OR 1.88; $1.56-$ 2.28), were significantly more likely to seek care at the SHC. Students with symptoms were more likely to seek care at the SHWC (OR 0.53; 0.47-0.61), while persons who reported contact with STIs were more likely to seek care at the SHC (OR 2.88; 2.22-3.74). The overall percentage of positive screenings was $9.3 \%$ for chlamydia (CT), 3.0\% for gonorrhea (GC), $0.8 \%$ for trichomoniasis (TV), $0.7 \%$ for syphilis, and $0.3 \%$ for HIV with higher percentages of positive for CT (OR 1.60; 1.30-1.96) and GC (OR 2.02; 1.44-2.85) in the SHC.

Conclusion Embedding a dedicated SHC within a university SHWC may expand populations reached for STI screening. With higher percentages of patients testing positive for CT and GC, a SHC may allow for greater diagnosis and treatment of STIs in general screening and persons presenting as contacts.

\section{P359 OPTIMIZED METHODS TO MODEL MYCOPLASMA GENITALIUM REPRODUCTIVE TRACT INFECTION IN PIG-TAILED MACAQUES}

L Aguila*, D Patton, G Wood. University of Washington, Seattle, USA 